\title{
Italique
}

Poésie italienne de la Renaissance

XVII | 2014

Varia

\section{Per una rilettura delle Rime di Messer Luca Contile}

\section{Ester Pietrobon}

\section{OpenEdition}

\section{Journals}

Edizione digitale

URL: http://journals.openedition.org/italique/395

DOI: $10.4000 /$ italique.395

ISSN: 1663-4438

\section{Editore}

Librairie Droz

\section{Edizione cartacea}

Data di pubblicazione: 1 ottobre 2014

Paginazione: 207-227

ISBN: 978-2-600-01841-8

ISSN: 1423-3983

\section{Notizia bibliografica digitale}

Ester Pietrobon, «Per una rilettura delle Rime di Messer Luca Contile », Italique [Online], XVII | 2014, online dal 01 octobre 2016, consultato il 19 avril 2019. URL : http://journals.openedition.org/ italique/395 ; DOI : 10.4000/italique.395 


\section{Ester Pietrobon}

PER UNA RILETTURA DELLE

RIME DI MESSER LUCA CONTILE 



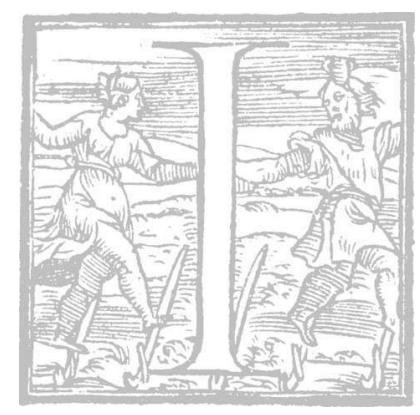

$n$ un recente intervento per il seminario di studi su Luca Contile svoltosi nel 2007, Domenico Chiodo ha richiamato l'attenzione degli studiosi sul libro di Rime del Is60, individuando la corda peculiare di questa poesia nell'ispirazione encomiastica e cortigiana che egli sintetiza efficacemente nell'immagine della «musa in livrea». ${ }^{\mathrm{I}}$ Nel tornare a scrivere oggi di quest'opera, ci proponiamo come obiettivo fondamentale un'analisi più dettagliata del libro, seppur condotta per campioni esemplari, che miri a porre in evidenza gli elementi di coesione a livello macrotestuale da un lato, $\mathrm{e}$ i diversi fattori di interazione sinergica fra $i$ testi lirici e le relative prose esegetiche di Francesco Patrizi e di Antonio Borghesi dall'altro. Per raggiungere una valutazione quanto più obiettiva possibile della qualità dei sonetti contiliani, lontano da facili entusiasmi euristici o da troppo severi giudizi come quelli espressi da Abd-el-Kader Salza all'inizio del secolo scorso, ${ }^{2}$ cercheremo di ricostruire gli equilibri della raccolta in una visione d'insieme che non trascuri il tutto per concentrarsi sulla parte (o sulla Parte, dovremmo dire, giacché l'attenzione della critica si è sempre, più o meno a ragione, appuntata in modo esclusivo sulla Parte prima commentata da Patrizi) e che rilevi infine come la giusta ottica per una comprensione piena sia del testo poetico sia delle diverse operazioni ermeneutiche si confermi ancora una volta quella di una contestualizazione dinamica e attenta all'intero sistema del Testo ${ }^{3}$.

Le rime di MesserLuca Contile divise in tre parti, con discorsi, et argomenti di M. Francesco Patritio, et M. Antonio Borghesi. Nuovamente stampate. Con le sei canzoni dette le sei sorelle di Marte ${ }^{4}$ sono una raccolta composta da tre sezioni sonettistiche la prima con so sonetti d'argomento amoroso, commentata da Patrizi; la seconda con 53 sonetti in morte del Marchese del Vasto Alfonso d'Avalos e la terza, piu ampia, con 83 sonetti d'occasione e di corrispondenza e una canzone dedicata a Camilla Pallavicini, entrambe commentate da Borghesi - e conclusa dalla ristampa delle sei canzoni politiche già edite nel I556, dedicate ciascuna a un capitano di parte imperiale, tra $i$ quali figura, ultimo ma non ultimo, lo stesso Filippo d'Asburgo. Infine compaiono due sonetti, anch'essi corredati 
dai relativi argomenti, che riportano $i$ numeri LXXXIIII e LXXXV: si tratta di due omaggi in mortem rispettivamente a Irene di Spilimbergo e a Claudio Tolomei, inseriti all'ultimo nella raccolta.' Questa pubblicazione di chiaro stampo encomiastico rappresenta per il cortigiano Contile uno strumento utile ad attirarsi la benevolenza non solo dei signori d'Avalos, ma soprattutto di Filippo II di Spagna, con la speranza, rimasta delusa, di giungere a prestare servizio presso la stessa corte imperiale. È interessante ricordare con Daniele Ghirlanda ${ }^{6}$ che l'edizione del '6o nasce come una deviazione dal progetto originario dell'autore di allestire un canzoniere onnicomprensivo delle proprie rime: il ms. angelicano 2407, infatti, mostra che in una fase di revisione, culminata in una seconda redazione del testo, Contile espunge mediante asterisco tutti $i$ sonetti poi inclusi nelle Rime, eccezion fatta per il numero XXI. La raccolta angelicana si configura quindi, dopo il Is60, in senso più specificamente spirituale, assumendo la forma di un canzoniere dalla struttura ben delineata, corredato da un autocommento lasciato però incompiuto.

Se il movente encomiastico della silloge veneziana è palese $-e$ in questa spinta derivante dalla "ragion pratica" dell'uomo di corte va riconosciuto il motivo ideale che lega e anima l'intera raccolta - risulta più arduo stabilire la pertinenza, dal punto di vista formale, di una possibile definizione di canzoniere invece che di semplice libro di rime. Secondo Gorni, è possibile parlare di «canzoniere» ogni qualvolta ci si trovi di fronte a un libro di poesia «in cui sia evidenziabile, a uno o più livelli del testo, qualche intento di organizzazione interna della materia». ${ }^{7}$ Tuttavia, se andiamo a confrontare le Rime cristiane con le Rime del '6o, ci accorgiamo di quanto la dispositio dei testi sia in realtà molto più ragionata e rigorosa nella raccolta manoscritta, ${ }^{8}$ mentre nel secondo caso l'elemento portante della struttura del libro non sembra essere quello interno al testo - anche se non mancano meccanismi di concatenatio tra $i$ singoli sonetti - ma sia piuttosto da individuare nel paratesto costituito dal commento. La prosa esegetica funge dunque da fattore modellante del liber, che in un quadro complesso e denso di riflessioni sul senso della poesia e sulla forma da dare a una lirica autenticamente nuova come quello della Venezia del secondo Cinquecento, ${ }^{9}$ si richiama in maniera implicita a modelli formali alternativi quali l'Orazio lirico $(50+53$ sono $i$ sonetti delle prime due parti, tanti quanti le Odi del Venosino, così come la tripartižione è ascrivibile alla 
stessa matrice oraziana) e rivendica in aggiunta, almeno per $i$ cinquanta sonetti d'amore dedicati a Giovanna d'Aragona, una novità contenutistica rispetto a Petrarca. ${ }^{10}$ È necessario, però, considerare con cautela tutti questi elementi per non cadere nell'errore di sovrastimare la portata filosofica, e di conseguenza la qualità poetica, dei sonetti commentati da Patrizi, svalutando per contro gli altri componimenti esposti da Borghesi. La diversa caratura dei due commenti, infatti, non corrisponde a un'effettiva differenza di valore tra $i$ sonetti delle varie sezioni, ma indica semmai due tipologie di approccio al testo molto distanti tra loro: una di carattere filosofico-retorico, a sfondo sapienziale; l'altra rivolta alla semplice chiarificazione del testo e della sua occasione, senza ulteriori pretese interpretative.

Mentre le peculiarità e, sen za dubbio, le novità dell'apparato ermeneutico del Chersino sono state da noi approfondite in altra sede, ${ }^{\mathrm{II}}$ è interessante ora soffermarsi sul corpus lirico delle Rime, individuando le costanti tematiche e lessicali che uniscono $i$ sonetti delle tre parti, senza dimenticare che in un'edizione come questa, dove il testo lirico viene pubblicato per la prima volta già provvisto di un supporto esegetico, non si può mai prescindere dalla complementarietà statutaria dei due elementi. Ricordiamo, inoltre, che il commento - pratica assai diffusa in primis sul Petrarca volgare e poi estesa, almeno a livello progettuale, a tutta la «biblioteca» dei grandi autori greco-latini e volgari italiani - ha il duplice scopo di legittimare la poesia contiliana nell' affollato panorama lirico di metà secolo e, soprattutto, di presentare Contile come un classico a tutti gli effetti: ${ }^{2}$ non a caso, seppur con accenti evidentemente faziosi, Patrizi dichiara che nei suoi cinquanta sonetti amorosi l'amico supera, per "concetti», lo stesso Francesco Petrarca. ${ }^{{ }^{3}}$ La «lode»è dunque la nota dominante della raccolta: Patrizi la indica nei suoi Argomenti come il «concetto, o intendimento» principale dei sonetti per donna Giovanna, ma quest'affermazione può essere estesa in ultima analisi anche ai componimenti delle altre sezioni. La lode che nasce da amore, infatti, non ha un carattere cosi diverso dall'encomio rivolto al Marchese del Vasto o ad altre personalità della corte, poiché sorge dalla medesima volontà celebrativa che giustifica in maniera ben più convincente rispetto alla lettura neoplatonica patriziana la frigidità di questi sonetti. ${ }^{14}$ Il campo metaforico piu frequentato per la tessitura dell'elogio cortigiano è senz'altro quello della luce, con una predilezione per l'immagine, in realtà assai convenzionale, del sole come espressione 
di massima luminosità, e quindi di altezza e di «eccellenza», nobiliare e d'animo, dei destinatari. Si confrontino a questo proposito $i$ sonetti proemiali della prima (I, I, c. Ir) e della seconda parte (II, I, C. 38 r):

Qual occhio che nel Sol perde la vista

A sé debil ritorna, e nulla vede,

Onde ne l'alte e luminose prede

Del suo picciol valor tutto s'attrista;

Tal è la mia vertù, mancando, trista

Nel chiaro oggetto ch'ogni lume eccede,

Onde nasce bellezza, e dove riede:

Ch'in Cielo, in terra no, tal don s'acquista.

Dunque lodar potrà lingua mortale

Bellezza eterna, sì ch'a par s'intenda

Alto splendor per basso stil terreno?

Io cantarò, ma (desir mio) che vale,

(Se non è che per gratia Dio n'accenda)

Breve favilla presso al Ciel sereno?

La vostra alma vertute è puro e fermo

Splendor di bene, e d'alta gloria essempio

Privation d'affetto amaro e scempio

E contra ogni atto vil libero schermo.

Indi prendo io come mortale e infermo

Norma di mia salute, indi il cor empio

De' cristiani concetti fassi tempio

Quivi il Sol sete, ove io la vista fermo.

Poi mi rivolgo al primo Sole, onde hebbe

Principio il vostro, e gratia di far lume

$\mathrm{Al}$ tenebroso mio sviato core.

E dico, o quanto il secol nostro debbe,

A te beato e glorioso nume

Poiché Alfonso c'insegna a farti honore.

Il comparante del sole viene utilizzato sia per Giovanna d'Aragona (I, I, I) che per Alfonso d'Avalos (II, I, 8) oltre che, naturalmente, per Dio, invocato con una probabile reminiscenza dantesca di Inf. III $5-6^{15}$ come «primo Sole» (II, I, 9). Si noterà che, mentre la vista della donna amata (il «chiaro oggetto ch'ogni lume eccede, / Onde nasce bellezza, $e$ dove riede») provoca nell'amante l'accecamento, privandolo cosi della 
facoltà visiva ma aprendogli la strada della visione spirituale e beatificante (anche se qui, in realtà, prevalgono $i$ topoi dell'ineffabilità della «bellezza eterna» di Madonna e dellinadeguatezza del poeta a cantarla degnamente), il cortigiano Contile riesce a fissare gli occhi sul soleAlfonso ("Quivi il Sol sete, ove io la vista fermo») che costituisce la via per accedere alla visione più alta di Dio, «principio» di tutte le cose e quindi anche dello stesso marchese, che insegna al cuore «tenebroso»e «sviato» del poeta ad onorare il «beato e glorioso nume». Il sintagma «tenebroso core» richiama la «tenebrosa mente» del sonetto IX della prima parte, dove Contile paragona lo sguardo della sua donna ai raggi solari e afferma che come il sole penetra e rischiara con la sua luce tutto l'universo, cosi gli occhi di lei illuminano la sua mente ottenebrata (I, IX, $I-8, c .3 r):$

Come il Sol del suo Dio vivo occhio ardente, Tanti infiniti corpi, in tanti cieli Penetra col suo lume, onde riveli Ciascun più bello il suo stato, e lucente;

Tal nella mia sì tenebrosa mente

Passan le vostre luci, e d'ombre e veli

La sgombran sì, che da fumi e da gieli

Giamai non foran maculate, o spente.

Appare evidente, a questo punto, come la donna e il signore rivestano entrambi la medesima funzione di via salutis tanto cara non solo al neoplatonismo ficiniano, ma anche alla lirica stilnovista e allo stesso Petrarca.

Si confrontino ancora l'espressione «splendor di bene, e d'alta gloria essempio» che descrive il valore di Alfonso e l"«alto splendor» di donna Giovanna: se nel primo caso la tematica dell'«essempio» richiama l'illustre precedente petrarchesco di $\operatorname{Rvf} C L I X,{ }^{2}-4,{ }^{16}$ lo splendore della bellezza di madonna implica senza dubbio un rimando a Ficino. Al di là delle connotazioni salvifiche e filosofiche, però, la luce divina che si irradia dai marchesi è pur sempre quella della causa per cui Contile agisce e per la quale si spende con tutte le sue forze sia nell' attività di segretario, sia nell'encomio dei suoi signori.

Se nella sezione amorosa la similitudine astronomica appare maggiormente connotata in senso filosofico e talvolta, a detta di Patrizi, anche ermetico-cabalistico, nei sonetti successivi essa assume, invece, toni elo- 
giativi di puro carattere occasionale, come anche sfumature salvifiche d'impronta cristiana. Queste oscillazioni semantiche della figura luminosa non pregiudicano, tuttavia, la sua natura fondamentale di topos encomiastico che rimane costante in tutta la raccolta. Si osservi, inoltre, come il linguaggio e le topiche d'amore diventino elementi costitutivi della lode del cortigiano, trovando cosi un'applicazione ulteriore rispetto all'ambito della lirica d'amore tout court. Contile stesso descrive nei termini dell'innamoramento il proprio rapporto con due signori, il marchese Alfonso d'Avalos e il cardinale Trivulizio, in una lettera del 29 luglio $154 \mathrm{I}^{\mathrm{I7}}$

\section{Al Marchese del Vasto.}

Ho havuta gran commodità di saper, e di veder con gli occhi stessi il modo smanioso, e non amoroso, di quello che per gelosia conduce V. Eccel. a dar terrore a gli innocenti. Ho conosciuto per la parte de l'innamorato ch'egli è degno di compassione, non in quanto a l'amore, che s'attacca sempre a cuor gentile, ma sì bene in quanto a la cagione. Né mi so imaginare onde habbia origine questa monstruosità, essendo necessariamente vero che 'l lume procede da la luce, che la bontà nasce da la Vertù, che l'huomo è prodotto e generato da l'huomo, e la bestia da la bestia. Amore non procede da l'odio; Amore non ha veramente altro luogo, et altro recettacolo, che la bellezza. Le passioni amorose non vengono altronde che dal desiderio di bellezza, e 'l desiderio non si riposa, o non fa mai riposare lo Amante fin tanto che non possiede la cosa amata, che altro non è che la bellezza, e la bellezza non solamente diremo esser in un bell'occhio, in un bel viso, in un bellissimo, e acceso colore de le guancie di bella, vaga e dilettosa Donna, d'intorno a la quale splendin le tre gratie, e nella letitia de l'aspetto, e nella verdura de l'età giovanile, e nel piacere de' moti e gesti del corpo; ma ancora nelle attioni, e nelle opere, ove si fanno chiare e visibili le vertù de l'animo. E per esser costei vecchia, magra, scolorita, con gli occhi di pappagallo, con bocca larga, di aspetto crucioso e disgratiato, senza alcun diletto di ciò che in essa si vede, e poi di nome non so come, che diremo adunque ch'ad amarla conduca quel Satrapo, se non interesse di robba, o bassezza di cuore? Potrebbe forse esser cagione di questa monstruosità la conferenza di sangue, ma che per questo si chiami Amore, non si può comportare. Iddio ne scampi da simile disgratia tutti gli huomini da bene. ${ }^{18}$ 


\section{A questo punto risulterà più chiara la convergenza tematica tra sonetti} come $i$ seguenti, che riportiamo insieme ai relativi argomenti:

Quanto sia degna l'alma di costei,

Lo mostra il corpo e le sue parti belle,

Che come il ciel adorno è d'alte stelle,

Di cotai lumi Dio tien ricca lei.

Io già miglior essenza non vorrei

Poich'amor mi fa degno d'amar quelle

Bellezze, onde convien ch'io ne favelle

O che manchin di moto i giorni miei.

E come per vedere il Sol, conviene

Guardar i luoghi ove ei percote tanto

Il suo splendore, ch'i mortal' occhi avanza.

Così vegg'io la donna, onde mi viene

$\mathrm{Al}$ cor sì bello essempio, e mentre io 'l canto

La segue un santo ardor fuor di speranza. (I, XXxIII, c. 9r)

Il sogg. è la bellezza dell'anima. È accresciuta la lode dal luogo de' conseguenti. Percioché consegue quasi di necessità che bell'anima sia accompagnata da bel corpo. Il quale sia come testimonio della bellezza di lei. E dalla comparatione del cielo stellato, dallo effetto cagionato in lui, e dalla simiglia de' percotimenti del Sole. Che sono testimonij della sua presenza. Né questo è nel Petr. (c. 33v)

Amici, io bramo ogni hora, ogn'hor vorrei

Goder come co 'l cor, con gli occhi tanto,

Il Signor nostro, in cui mi pregio e vanto,

Ch'egli sia il lume de' concetti miei.

E se mi sono i fati acerbi e rei

Sì che io depongo, mio malgrado, il canto;

Pur le sue qualità celebro e canto

Con quel pensier che scriver non saprei.

Ma quando a me medesmo lieto narro

Del Signor nostro la gran meraviglia

Io n'ho invidia al Carpegna, invidia a Lillo.

E se no fosse l'humido catarro

Ch'al cor mi cala da le fosche ciglia,

Havrei il tempo con voi lieto e tranquillo. (II, XXI, c. 43r) 
Era ammalato di catarro l'Aut. quando il Marchese lo volle menare a Vegevene, dimostrando che se bene non era dove il suo signore, scrivendo al Conte Hettorre di Carpegna et al Cavalier Vergilio, non dimeno lo haveva nel pensiero sempre, concludendo che gran contentezza havrebbe havuto con loro in vederlo. (c. $54 \mathrm{r}$ )

Pur con le inevitabili differenze di tono, colpisce la ricorrenza di luoghi comuni tanto alla lode della donna - nonché domina, secondo la duplice accezione amorosa e cortigiana - quanto all'encomio del marchese. Innanzitutto, come si è notato in precedenza, il poeta attiva nei confronti di entrambi gli oggetti della sua contemplazione il binomio già petrarchesco di «vista» e «visione» («occhi» e «con»), esprimendo nel secondo sonetto l'idea dell'inadeguatezza della propria poesia che ritroviamo, solo per citare un esempio, anche nel sonetto d'apertura della prima parte. Alfonso è chiamato «lume de' concetti miei» con una sfumatura più neutra rispetto al paragone di temperie ficiniana tra Giovanna e il sole: la donna, infatti, non è una semplice fonte d'ispirazione, ma funge da tramite tra Dio e il poeta, giacché a quest'ultimo, a cui è preclusa la visione diretta dell'astro (comparante della divinità) per il suo eccessivo «splendore», è possibile soltanto una visione indiretta che avviene fissando lo sguardo sui «luoghi» illuminati dal sole. Uno di questi luoghi è appunto la donna, che attraverso gli occhi imprime un "essempio» di sé stessa nel cuore dell'amante e gli permette cosi di poetare e di avvicinarsi mediante quest'esercizio a Dio.

Degno di attenzione è, infine, iltermine «meraviglia» (II, XXI, IO), usato da Contile in riferimento al marchese. Si ricordi che Patrizi, nel suo primo argomento, parla della meraviglia come dell'effetto prodotto dalla bellezza nell'anima di chi la osserva, il quale a sua volta è in grado di generare «Amore» nel cuore oppure «lode», cioè esaltazione della «virtù» e dell' «altrui eccellenza», espressa oralmente ( $" s$ su la lingua») o in poesia ( $" s u$ la penna»). La facoltà poetica nasce dunque da qualcosa di miracoloso che solo la bellezza è in grado di originare un invasamento - un furor - che comporta allo stesso tempo un'implicazione della «ragione» intesa come «potenza conoscente», sicché la formulazione della «lode»presuppone una presa di possesso conoscitiva della bellezza, che diventa cosi insieme «oggetto» del pensiero e «soggetto» della poesia.

Ma perché la bellezza, qual si sia, partorisce sempre maraviglia nell'animo di colui che la vede e la conosce, et la maraviglia sempre genera o nel 
cuore dell'ammiratore Amore, o lode su la lingua e su la penna; prende l'autore per principale suo concetto, o intendimento, in tutti questi sonetti, la lode. La quale è un parlare dimostrante la grandezza dell'altrui virtù, disse Aristotile; et, io dico, l'altrui eccellenza. Ma perciò che tutto ciò che è formato dalla penna, o dalla lingua, è prima formato nel pensiero; et il pensiero è opra della ragione; e la ragione è potenza conoscente; et ciò che ella conosce, è oggetto; et conosce hora la ragione dell'autore la perfetta bellezza di Donna Giovanna; la bellezza adunque di questa Donna è et oggetto del pensiero dell'autore, et soggetto della presente lode di lei. (c. $25 \mathrm{v}$ )

Esiste quindi un punto d'incontro profondo nell'ispirazione e nel linguaggio di questi testi che, tuttavia, Patrizi non sembra cogliere, o voler cogliere. Il critico si dimostra intento alla ricostruzione di un ponderoso e talvolta pletorico sostrato filosofico che caratterizzerebbe $i$ sonetti della prima parte, allontanandosi però troppo spesso dalla realtà testuale e, soprattutto, trascurando il passaggio fondamentale della rifunzionalizzazione encomiastica del lessico amoroso, che costituisce una chiave determinante per la comprensione di questa poesia. Se $i$ sonetti per donna Giovanna presentano naturalmente dei tratti ficiniani, riconducibili peraltro ad una solida e riconosciuta competenza in tal senso dell'autore, non bisogna cadere nell'errore prospettico di attribuire a tale dato una rilevanza assoluta, a scapito di quanto emerge da un esame più completo che tenga conto del contesto generale della raccolta.

Riportiamo ancora un sonetto della seconda parte, dove Contile celebra il ritorno della marchesa del Vasto. L'autore si rivolge a Isabella Brivia, nobildonna di corte alla quale aveva già dedicato un altro sonetto, per celebrare le lodi della sua signora. Si osservi, accanto alle consuete iperboli a carattere luminoso, l'ulteriore ripresa a fini encomiastici del tema della donna come via salutis: la "luce che ogni hor move / gli animi al bene» è quella che «insegna a fruir l'amor celeste / che sol gli spirti dolcemente lega, / e mille beni in un sol punto mostra». Si tratta di un segnale importante che chiarisce meglio anche la natura dei primi cinquanta sonetti, dimostrando ancora una volta l'unità di intenti del libro di rime.

Brivia, torna la luce che ogni hor move

Gli animi al bene, al santo oprar gli ingegni,

Da la cui vista in noi nascon disegni, 
Di meraviglie rare e gratie nove.

Felice loco, ove ella posa, e dove

Nudre chi merta, e schifa gli atti indegni,

Accresce la pietà, placa gli sdegni,

E ci fa grati a Marte, a Febo, a Giove.

Indi insegna a fruir l'amor celeste

Che sol gli spirti dolcemente lega,

$\mathrm{E}$ mille beni in un sol punto mostra.

Ecco, dico, la luce che riveste

Il bel Sol de' suoi raggi, e con lui spiega

L'ale di pace a la fortuna nostra. (II, XIX, c. 42V)

Scrive il decimo nono alla S. Isabella Brivia, alla quale scrisse ancora il decimo. Il soggetto è la letitia del ritorno da Napoli a Milano della Sig. Marchesa, la quale con vertuosi e degni attributi è da lui laudata, concludendo che la stessa Sig. è la quiete della fortuna de' suoi servitori et amici. (c. $54 \mathrm{r}$ )

Si leggano ora alcuni testi della terza parte. Il sonetto proemiale della sezione ribadisce con forza le posizioni filospagnole di Contile: il poeta celebra le virtù ritrattistiche del cavalier Leone - lo stesso a cui aveva dedicato il Discorso sopra li cinque sensi del corpo nel I5s $2{ }^{19}$ e, in particolare, elogia una medaglia d'oro da lui scolpita con l'effigie del «Serenissimo Re Catholico» Filippo II di Spagna. Le «luci interne» dell' «invitto Re» sono manifestate nella «piastra d'oro», che diventa cosi emanaz̧ione della regalità e insieme originale reinterpretazione del tema luminoso in chiave encomiastica.

Sembra il real disegno Apollo e Marte,

Anzi in quel l'uno e l'altro si discerne

$\mathrm{E}$ fansi in ciò mirabili et eterne

La vista, l'aria, la materia e l'arte.

Né pur si mira quivi alcuna parte

Che dello invitto Re le luci interne

Non scopra, onde l'antiche e le moderne

Forme per questa havran men chiare carte.

Fu sol la vostra mano, e 'l vostro ingegno

(Questa maravigliosa e quel divino)

C'han sì gran Re scolpito in piastra d'oro. 
Voi beati a portar sì ricco pegno,

Sempre co 'l cor devoto e 'l capo chino,

Riverite di Dio tanto tesoro. (III, I, c. 6or)

In questa terza parte de' sonetti del prefatto Autore brevemente s'accenna la sostanza de' suoi nobili e vertuosi pensieri et veramente mirabili concetti. Là ove nel primo scrive quanto sia la vertù dell'Arte del ritrarre del Cavalier Lione, scultore celebratissimo massimamente in medaglie havendo ritratto il Re Filippo Re di Spagna del proprio, concludendo che beati sono coloro che la portano, tesoro reverendo di Dio il quale ha dotato di tanta gratia il detto Serenissimo Re Catholico. (c. 83 r)

Una variazione sul tema del ritratto dorato si trova nel secondo sonetto, dove l'autore esalta le capacità ritrattistiche dello stesso Filippo e mette a confronto le due immagini fictae nell'oro della medaglia e nel cuore del capitano Marcantonio Spinola: il segreto per farle coincidere, dice il poeta, sta ancora una volta nell" «amore» e nella «riverenza》 che caratterizzano ogni buon cortigiano, il quale, come un vero e proprio amante, dovrà consacrare al proprio signore «l'opre, e la vita».

L'imagin bella (Spinola cortese)

Che da la real man fu in oro spressa,

Con quella che nel core havete impressa,

Vuol far (cred'io) dolcissime contese.

Dice d'oro la effigie: «Quali imprese

Fian mai simili a me? se da me stessa

Fatta mi sono? e se natura ha messa

In me sé propria, e me seco comprese?»

Ma l'altra che nel cor lieto tenete

Par che dica che amore e riverenza

Nel vostro vivo cor l'hanno scolpita.

E se pure accordarle ambe volete

E farle d'una vista e d'una essenza

Sacrate al vero Re l'opre, e la vita. (III, II, c. 6ov)

Nel secondo scrive al Sig. Marcantonio Spinola Capitanio della guardia del Re di Boemia, lodando la medaglia che egli porta in seno non solamente rappresentando il suo Re, ma la vertù che 'l detto Re ha in ritrarre di sua mano e del proprio, mostrando la quistione che può nascere fra il ritratto e quella che ha nel cuore scolpita, e conclude che le ridurrà pacifiche, quando perseveri fin alla morte, di servire all'istesso Re. (c. $83 \mathrm{r}-\mathrm{v}$ ) 
Interessante è poi il sonetto $\mathrm{V}$ per la marchesa Camilla Pallavicini, costruito sul tema della visione beatificante della donna, grazie alla quale il poeta contempla le «bellezze interne» di lei e riconosce in esse le immagini delle bellezze «sempiterne» $e$ «d'ogni ben gli amati fini». Il tono è con evidenza vicino a certi sonetti della prima parte, nei quali le lodi della donna amata e della signora riverita si fondono e si svelano reciprocamente.

Quanto opra il ciel ch'a voi più s'avicini

La vista mia, più par ch'in voi s'eterne

$\mathrm{E}$ con più suo piacere in voi discerne

Le bianche perle, e i splendidi robini.

E se fia che 'l mio spirto non dechini

Dal contemplar vostre bellezze interne,

Vedra.lle imagin de le sempiterne

E vedrà d'ogni ben gli amati fini:

Pur che le vostre vertuose e belle

Luci diano al mio stile e lume e senso

Che con tal scorta degne cose intendo.

Ma che temo io, se dal cor vostro quelle

Vertù m'han sì di santo foco accenso

Ch'in celebrarvi ogni mio ben comprendo. (III, v, c. 6rr)

Usa nel quinto la maestria delle medesime rime desinenti lodando la bellezza de l'animo e del corpo di quella honoratissima Sig. et dove nello antecedente dice che chi l'ascolta lo fa rimanere acceso per riverirla, così nel seguente mostra che quanto egli più la celebra, più conosce il suo proprio bene. (c. $83 \mathrm{v})$

Il sonetto seguente nasce, invece, da un'occasione simile a quella del sonetto IIII della seconda parte per la marchesa di Sonzino. L'autore si scusa con una gentildonna della casa di Giovan Battista Castaldo per averla confusa con una domestica, sfoderando a scopo riparatore tutta la ormai ben nota terminologia luminosa. La donna è definita «bel Sole» $e$ «lume altero», mentre Contile accusa la propria vista di non aver riconosciuto questa viva immagine della «fausta beltà»; il motivo è che $i$ «raggi de' begli occhi» di lei hanno abbagliato lo sguardo del poeta, uccidendo in questo modo anche l' "Idea» che egli aveva nel cuore. E' quasi superfluo sottolineare come elementi topici della lode amorosa già ricorrenti nella prima parte - si pensi alla luce della donna e ai raggi 
emanati dai suoi occhi - si pieghino qui meno che mai ad una possibile interpretazione in chiave filosofica: segno che forse, nonostante la differenza che sicuramente separa queste rime da quelle per Giovanna d'Aragona, la costruzione del Discorso patriziano non trova una piena giustificazione.

De la fausta beltà la viva imago

Ch'entro il mio petto ha vertuoso Impero,

Come non seppe alhor scernere il vero

Più che mai bello e dolcemente vago?

Perché non fosti del bel Sol presago

Spirto confuso? e come il lume altero

Non conoscesti? ahi folle mio pensiero

Di qual difesa tanto errore appago?

Vista mia cieca a sì bel Sol simile,

Chi festi? un'ombra? hor non vorrò più mai

Fidarmi già di te perfida e rea.

Di me non ti doler, fido Contile,

Ch'a raggi de' begli occhi m'abbagliai

E rimase nel cor morta l'Idea. (III, XLVII, c. 7 IV)

Occorse a l'Aut. ritrovandosi in Casa del S. Giovan Battista Castaldo in Milano di non conoscere la Signora Faustina Maggiolina Nuvolona, tanto sua domestica et honorata Sig. dove che di questa confusione fe' rimanere stupido ogn'uno, e quasi sdegnata quella vertuosa gentildonna, sopra tal caso scrive il presente son. concludendo con molta laude di essa Sig. la sua legittima scusa. (c. $89 \mathrm{v}$ )

Un altro tema connesso con la topica luminosa e che ricorre in tutte le sezioni è quello della fama. In questo caso, l'oggetto della lode di Contile è l'imperatrice Margherita d'Austria, le cui virtù «chiare e belle» sono paragonate all" «ampio lume» irradiato dalle innumerevoli stelle del cielo.

Ibero, Tebro, Reno, Istro più volte

Sentito ho risonar, donna, di voi

$\mathrm{E}$ dir che dei più cari culti suoi

Son dal vostro splendor le glorie tolte.

Quinci ben mille voci in una accolte

Da' Regi alzate al cielo, e d'altri heroi, 
Rimbombar fanno in dolci accenti a noi

Quante in voi sian di Dio gratie raccolte.

Come del ciel non numera le stelle

Saggio intelletto, ma pur d'esse vede

Sparso per sì gran cerchio un ampio lume,

Tal son le vertù vostre chiare e belle

Ch'infinite al pensier nostro fan fede

Che siate eterno, ma visibil Nume. (III, XVII, c. 64r)

Scrive il decimo settimo a l'Eccell. di Madama Margarita d'Austria mostrando che ella è lodata quasi per tutta Europa, e meritamente poiché ogni lingua a gara esalta le virtù di tanta Sig., né se le può dar maggior laude poiché ella è chiamata un Dio visibile. (c. $85 \mathrm{v}$ )

Altrove la stessa Margherita è chiamata «luce prima» d'Italia e la sua partenza è paragonata implicitamente alla scomparsa del sole: l'imperatrice lascia nelle «tenebre» il «bel nido» delle sue città italiane tanto che, fino al suo ritorno, Piacenza sarà un «tenebroso inferno» e il Po un «amaro vaso» per raccogliere le lacrime del popolo e della corte.

O di tanti in Italia luce prima,

Ch'in tenebre lasciate il lor bel nido

Nel partir vostro, onde mandran lo strido

Da questo al freddo et al cocente clima;

Che più s'apprezza homai, che più si stima

Nel nostro già sì fortunato lido?

Ahi fato iniquo tanto, ahi mondo infido

Cagion che 'l proprio danno io canti in rima

Le sacre Muse che in Italia ferno

Scorger novo Helicon, novo Parnaso

Quando appariste voi Cesarea Diva.

Fatta è Piacenza tenebroso inferno

E fatto è 'l Po di pianto amaro vaso,

Poiché di tanta luce Ausonia è priva. (III, XVIII, c. 64v)

Lecita cosa pare a l'Aut. che ciascuno si condoglia de la partita di Italia in Fiandra di Madama suddetta: perché veramente da la presenza di tanta donna il publico et il privato in tutta Italia ne riceve favore e giovamento, non è adunque gran fatto se egli conclude che fin tanto starà ella in Fiandra patiranno molti luoghi e particolarmente Piacenza e Parma. (c. 8 5v) 
Si è già accennato a un'ultima connotazione, quella spirituale, che la metafora luminosa assume in prevalenza nella seconda e nella terza parte, ma che non è del tutto assente nemmeno nella prima, se consideriamo l'insistita presenza del tema salvifico nei sonetti amorosi. Riportiamo soltanto, a titolo di esempio, due tra $i$ sonetti finali della terza sezione, nei quali la rilettura cristiana emerge in modo più deciso, non senza richiami più o meno espliciti alle dottrine neoplatoniche.

Nel sonetto LXXI Contile si rivolge a Domenico Venier per lodare le sue virtù, dichiarandolo uno dei pochi nomini degni di assurgere alla conoscenza di Dio. Il poeta afferma che la luce divina è nascosta in ogni nomo, poiché siamo stati creati a immagine di Dio, e traccia una scala ascensionale verso la divinità abbastanza simile al gradus amoris $d i$ platonica e ficiniana memoria. La differenza sostanziale, però, è che il percorso qui proposto è individuale ed è frutto di una rinuncia ad «ogni passion odiosa〉 del corpo in vista di una ricerca tutta trascendente del divino: siamo ben distanti, dunque, dalla teorizzazione del neoplatonismo radicale proposta da Patrizi. ${ }^{20}$

Poiché simili a Dio creati siamo,

Veniero, adunque è in noi sua luce ascosa;

Come però sì santa eterna cosa

Per vederla, e fruirla non cerchiamo?

Già se l'alma dal corpo dividiamo,

Da quella insieme ogni passion odiosa,

E da ragion la fantasia ritrosa

Da l'intelletto la ragion leviamo.

Poi se torrem da la sincera nostra

Mente de l'intelletto ancor le forme;

C'hanno dal mortal senso il primo grado,

Noi trovarem, pur come indora, e inostra

Lo Dio di foco in noi le sue sante orme,

E dove altro che tu s'erge di rado. (III, LXXI, c. 79v)

Ha voluto l'Autor nel presente sonetto mandar sì alta specolatione al Clariss. e dottissimo M. Domenico Veniero gentilhuomo di mirabile intelletto e di profonde scienze, sì come l'Autor stesso et ogniuno ampiamente ne parlano, accertandosi il detto Autore che tant'huomo, fra pochi, sia degno et atto a salire a gli stessi gradi della divina intelligenza. (c. $92 \mathrm{~V}$ ) 
Ecco, infine, l'ultimo sonetto della raccolta scritto da Contile in risposta a Filippo Binaschi. Borghesi ci informa che il nobile pavese, colpito da cecità durante gli anni giovanili, aveva chiesto al poeta di inviargli «alcune delle sue rime cristiane» affinché queste fossero oggetto delle sue meditazioni. Contile si schermisce e afferma che solo Cristo può condurre le anime a Dio. Quindi egli parla dell'infermità del suo corrispondente, effettuando per la circostanza una riformulazione cristiana del binomio vista-visione. La vista carnale è considerata una potenziale fonte di peccato, mentre la sua perdita rappresenterebbe un segno dello speciale favore di Dio che, in questo modo, permetterebbe a Binaschi di concentrarsi unicamente sul «lume» divino, facendo di lui un novello Argo «di vertì».

Binaschi, la mia trista e roca tromba

Da sé non può chiamar questa, e quell'alma,

Al bene eterno, e sol si dia la palma

A la pura di Cristo alta Colomba.

E se di trarti da la oscura tomba

Hai brama, come huom saggio, l'atra salma

Scorga la santa fé celeste et alma,

Ch'in ben purgato cor lieta rimbomba.

Di cotal lume a te Cristo comparte,

Poiché di quel sensibil ti diè poco,

Che a gli error nostri è sentier piano e largo.

Però con miglior voce a me sì fioco

$\mathrm{Fa}^{\prime}$ de' tuoi sacri e dolci accenti parte,

Tu de gli occhi orbo, e di vertù nuovo Argo. (III, LXxxIII, c. 82v)

Il presente son. è in risposta per quanto si vede, a uno che fu scritto a l'Aut. da M. Filippo Binaschi, gentilhuomo Pavese, il quale desiderava che 'l nostro Aut. gl'indrizzasse alcune delle sue rime cristiane, per le quali si sentiva disponere alla vita contemplativa, e felice. Usa modestia l'Aut. chiamandosi inabile et indegno e che Cristo solamente ci tira nel sentiero della beatitudine, essortando il Binaschi ad haver fede, la quale lo guidi: e perché lo stesso Binaschi, ancor che sia in questi nostri tempi huomo in tutte le scienze dottissimo, non dimeno è privo in tutto di vista e quasi da' teneri anni. Però l'Aut. gli dice che Cristo ha fatto parte a lui della sua gratia, Poiché gli ha tolto quel senso che ci conduce così agevolmente a peccare, e conclude che egli, più grato a Dio, è tenuto fargli parte delle sue sacre poesie: conclusione artifitiosa e poeticamente bella. (c. 94v) 
Anche gli spunti mistici tuttavia, al pari di quelli filosofici, vanno considerati in una posizione subordinata rispetto al fattore dominante dell'opera, ovvero al «disegno» spagnolo del cortigiano Contile. Non si dimentichi, infatti, che questi testi erano stati oggetto di una selezione precisa da parte del loro autore negli anni subito precedenti il Is 60 e che quindi, nel momento in cui erano stati scelti per essere inclusi in una raccolta encomiastica, erano stati anche implicitamente giudicati inadatti ad un progetto di canzoniere cristiano più genuino.

Ester Pietrobon 
I. Da questa metafora prende titolo l'articolo stesso: D. CHiodo, La musa in livrea: le Rime di Luca Contile, in Luca Contile. Da Cetona all'Europa. Atti del seminario di studi Cetona 20-2I ottobre 2007, a c. di R. Gigliucci, Roma, Vecchiarelli Editore, 2009, pp. I-Io.

2. Cfr. A.-E-.K SALzA, Luca Contile nomo di lettere e di negozj del secolo XVI. Contributo alla storia della vita di corte e dei poligrafi del 'soo, introduzione di A. QuONDAm, Roma, Bulzoni, 2007.

3. Sulla questione della forma-canzoniere e sulla struttura del libro di rime, a titolo di esempio e per la bibliografia pregressa, cfr. M. SANTAGata, La forma canzoniere, in M. Santagata-S. Carrai, La lirica di corte nell'Italia del Quattrocento, Milano, Franco Angeli, I993, pp. 31-39; Il libro di poesia dal copista al tipografo. Ferrara, 29-3 I maggio I987, a c. di M. Santagata e A. Quondam (in particolare F. Brugnolo, Il libro di poesia nel Trecento, pp. 9-23; A. Tissoni Benvenuti, La tipologia del libro di rime manoscritto e a stampa nel Quattrocento, pp. 25-33; G. Gorni, Il libro di poesia cinquecentesco: principio e fine, pp. 35-4I); S. Albonico, Sulla struttura dei "canzonieri" nel Cinquecento, in ID., Ordine e numero. Studi sul libro di poesia e le raccolte poetiche nel Cinquecento, Alessandria, Edizioni dell'Orso, 2006, pp. 29-46. Per la pratica esegetica e il rapporto tra testo e paratesto, cfr. Intorno al testo: tipologie del corredo esegetico e soluzioni editoriali. Atti del convegno di Urbino, I-3 ottobre 200 I, Roma, Salerno, 2003 (soprattutto L. BATTAglia Ricci, Comporre il testo: elaborazione e tradizione, pp. 2 I-40; F. BRugnolo- R. Benedetti, Testo e paratesto: la presentazione del testo fra Medioevo e Rinascimento, pp. 4I-6o; C. VILLA, I classici come modello, pp. 6I-75; S. CARrai, Il commento d'autore, pp. 223-4I).

4. Le rime di Messer Luca Contile divise in tre parti, con discorsi, et argomenti di M. Francesco Patritio, et M. Antonio Borghesi. Nuovamente stampate. Con le sei canzoni dette le sei sorelle di Marte, Venezia, Francesco Sansovino e compagni, i 560.

5. Chiodo, La musa in livrea cit., p. 7.

6. D. Ghirlanda, L'idea di un canzoniere: le Rime cristiane di Luca Contile, in Luca Contile cit., pp. I I-39, 24-29.

7. G. Gorni, Le forme primarie del testo poetico, in ID., Metrica e analisi letteraria, Bologna, I993, pp. i I 5-18. Cfr. inoltre la bibliografia di cui alla nota 3.

8. Cfr. l'analisi contenuta in GHIRLANDA, L'idea di un canzoniere cit.

9. Cfr. F. Tomasi, Le ragioni del 'moderno' nella lirica del XVI secolo tra teoria e prassi, in ID., Studi sulla lirica rinascimentale (I540-I570), Roma-Padova, Antenore, 201 2, pp. 3-24.

ı. Si veda quanto afferma Patrizi nel Discorso prefatorio alle Rime di Contile (Le rime di Messer Luca Contile cit., c. 23v): «Basta bene a' begli ingegni di accennare, che quanto sono più nobili i concetti de' più nobili amorosi godimenti, tanto è più nobile Poeta amoroso il Petrarca che qual si voglia de gli antichi, o Latini o Greci che essi sieno, essendo che egli mai non si abbassasse a dire quelle cose in che coloro hebbero posto tutti i desiderij loro. Ma quanta poi sia la 
differenza de' concetti del Contile in questi cinquanta amorosi Sonetti da quelli del Petr., si vedrà da chiunque vorrà quelli con questi comparare: il che io accennerò in ciascheduno».

i i. E. Pietrobon, Gli Argomenti di Francesco Patrizi come teatro ermeneutico del testo, in Canzonieri in transito. Lasciti petrarcheschi e nuovi archetipi letterari tra Cinque e Seicento, a c. di A. Metlica e F. Tomasi, Milano, Mimesis, in corso di stampa.

I 2. Per la pratica esegetica nel Cinquecento, oltre al già citato Intorno al testo, cfr. P. Petteruti Pellegrino, La negligenza dei poeti: indagini sull'esegesi della lirica dei moderni nel Cinquecento, Roma, Bulzoni, 2013.

I 3. Cfr. supra, nota io.

I4. Cfr. Chiodo, La musa in livrea cit., p. 8.

i 5. Inf. III 5-6: «Fecemi la divina podestate, / la somma sapienza e 'l primo amore».

I6. $R v f$ I 59, I-4: «in qual parte del ciel, in quale ydea / era l'exempio, onde Natura tolse / quel bel viso leggiadro, in ch'ella volse/ mostrar qua giù quanto lassù potea?»

17. Cfr. P. Procaccioli, Contile epistolografo. Le Lettere tra autopromozione e «speculazione de i perfetti modi, che usar si deono», in Luca Contile da Cetona all'Europa cit., pp. 297-344, p. 333.

i 8. Luca Contile, Delle lettere, Pavia, Girolamo Bartoli, i 5 64, libro i, c. 2 I r-v.

19. Discorso del Contile academico fenicio sopra li cinque sensi del corpo nel comento d'un sonetto del Signor Giuliano Goselini, al cavalier Leone scultore Cesareo, Milano, Valerio e Girolamo Meda, I 552.

20. Cfr. Pietrobon, Gli Argomenti di Francesco Patrizi cit. 